\title{
The existence of stationary star solutions for compressible magnetohydrodynamic flows
}

\author{
Huichao Wang ${ }^{1}$ and Ruikuan Liu, ${ }^{1,2^{*}}$
}

\section{"Correspondence:}

liuruikuan2008@163.com

'Department of Mathematics,

Sichuan University, Chengdu,

Sichuan 610064, P.R. China

${ }^{2}$ Department of Mathematics,

Northwest Normal University,

Lanzhou, 730070, P.R. China

\begin{abstract}
In this paper, we are concerned with the compressible Euler-Poisson system coupled to a magnetic field in the three-dimensional space. Based on a variational method and the exact expression of the Green's function for an elliptic equation in spherical coordinates, we prove the existence of stationary star solutions.
\end{abstract}

Keywords: Euler-Poisson system; magnetic field; variational method; stationary star solutions

\section{Introduction}

The Euler-Poisson system of compressible fluids coupled to a magnetic field is given by

$$
\left\{\begin{array}{l}
\rho_{t}+\operatorname{div}(\rho v)=0, \\
(\rho v)_{t}+\nabla \cdot(\rho v \otimes v)+\nabla p(\rho)=-\rho \nabla \Psi+\mu_{0}(\nabla \times H) \times H, \\
H_{t}=\nabla \times(v \times H), \\
\Delta \Psi=4 \pi G \rho, \\
\operatorname{div} H=0,
\end{array}\right.
$$

where $\rho$ is the density, $v=\left(v_{1}, v_{2}, v_{3}\right)$ is the velocity field, $H=\left(H_{1}, H_{2}, H_{3}\right)$ is the magnetic field, $p$ is the pressure function, $\Psi$ is the Newtonian potential, $G$ is the gravitational constant, and $\mu_{0}$ is the permeability of vacuum. We consider the polytropic gases for which the equation of state is given by

$$
p=p(\rho)=\rho^{\alpha},
$$

where $\alpha>1$ is the adiabatic exponent. The gravitational potential $\Psi$ is given by

$$
\Psi(x)=-\int_{\mathbb{R}^{3}} \frac{\rho(y)}{|x-y|} d y=-\rho * \frac{1}{|x|},
$$

where $*$ denotes convolution.

Extensive works have been done on the existence of stationary solutions for system (1.1) in [1-9] and references therein. Recently, some important progress has been made for system (1.1) without magnetic field. For the pressure $\alpha$-law in (1.2) with the adiabatic expo-

(c) The Author(s) 2016. This article is distributed under the terms of the Creative Commons Attribution 4.0 International License (http://creativecommons.org/licenses/by/4.0/), which permits unrestricted use, distribution, and reproduction in any medium, provided you give appropriate credit to the original author(s) and the source, provide a link to the Creative Commons license, and indicate if changes were made. 
nent $\alpha>\frac{3}{2}$, the global existence of weak solutions was obtained in [10] when the spatial dimension is three in the framework of Lions and Feireisl for the compressible Navier-Stokes equations [11, 12]. Furthermore, Cai and Tan [2] have proved the existence and uniqueness of stationary solutions of the three-dimensional compressible Navier-Stokes-Poisson equations basing on the weighted $L^{2}$ method and the contraction mapping principle.

For a nonrotating gaseous star, it is important to investigate the spherically symmetric motion since the stable equilibrium configuration is spherically symmetric. Moreover, the spherically symmetric solution minimizes the energy among all possible configurations [7], which are called Lane-Emden solutions. More importantly, Luo, Xin, and Zeng [13] were concerned with three-dimensional spherically symmetric solutions of the compressible Navier-Stokes-Poisson equations with free boundary condition. They also proved the nonlinear asymptotic stability of the Lane-Emden solutions for spherically symmetric motions of viscous gaseous stars if the adiabatic constant $\alpha$ lies in the stability range $\left(\frac{4}{3}, 2\right)$.

For the rotating nonmagnetic stationary solutions, Auchmuty and Beals [1] gave a priori bound for the maximum of the density $\rho$. When the rotation is a fixed axis with constant angular velocity, Chanillo and Li [3] obtained a priori bound for the support of the relative equilibrium form of a homogeneous, gravitating, and compressible mass of fluid. Coupling to the magnetic field, Federbush, Luo, and Smoller [4] first proved the existence of axisymmetric stationary solutions of system (1.1). They utilized a variational method and proved the existence of a stationary solution expressed by density, which is a minimizer of the associated energy functional. To prove the main result, an elliptic equation is derived for the magnetic potential in cylindrical coordinates in $\mathbb{R}^{3}$. Let $x=\left(x_{1}, x_{2}, x_{3}\right) \in \mathbb{R}^{3}, r=\sqrt{x_{1}^{2}+x_{2}^{2}}$, $z=x_{3}$. They looked for solutions of the following form:

$$
\left\{\begin{array}{l}
\rho(x)=\rho(r, z), \quad \Psi(x)=\Psi(r, z), \\
H(x)=H_{r}(r, z) e_{r}+H_{\theta}(r, z) e_{\theta}+H_{z}(r, z) e_{z},
\end{array}\right.
$$

where $e_{r}=\left(\frac{x_{1}}{r}, \frac{x_{2}}{r}, 0\right)^{T}, e_{\theta}=\left(-\frac{x_{2}}{r}, \frac{x_{1}}{r}, 0\right)^{T}, e_{z}=(0,0,1)^{T}$.

It is well known that almost every Newtonian gaseous star has crystal body. Auchmuty and Beals [1] have obtained a nonrotating nonmagnetic spherically symmetric solution of some nonlinear integro-differential equations in $\mathbb{R}^{3}$, which are of interest in astrophysics. Also, they formulated each problem as a variational problem and looked for a solution among an appropriate class of nonnegative functions $\rho$. In this paper, for the nonrotating magnetic case, we suppose that the stationary solutions should have the following form:

$$
\left\{\begin{array}{l}
\rho(x)=\rho(r, \theta), \\
H(x)=H(r, \theta)=\left(H_{r}(r, \theta), H_{\theta}(r, \theta), 0\right), \\
\Psi(x)=\Psi(r, \theta), \\
r^{2}=x_{1}^{2}+x_{2}^{2}+x_{3}^{2}, \quad \theta=\arccos \frac{x_{3}}{r} .
\end{array}\right.
$$

Motivated by the paper [4], we show the existence of stationary star solutions (1.5) of system (1.1) in spherical coordinates by using the variational methods.

The paper is organized as follows. In Section 2, we obtain an expression of stationary equations of system (1.1) in spherical coordinates by using the formula in [14]. Owing to the methods in $[7,8,15]$, we see that the stationary solution can be expressed by the density that is a minimizer of the corresponding the energy functional. Thus, we only need to derive the minimizer of the corresponding energy functional in Section 3. 


\section{Euler-Poisson system coupling with a magnetic field}

In this paper, we are interested in the stationary solutions of (1.1), which represent an important class of equilibrium configurations. The stationary solutions $(v=0)$ satisfy the following system:

$$
\left\{\begin{array}{l}
\nabla p(\rho)=-\rho \nabla \Psi+\mu_{0}(\nabla \times H) \times H \\
\Delta \Psi=4 \pi G \rho \\
\operatorname{div} H=0
\end{array}\right.
$$

where $\rho$ is the density, $H=\left(H_{1}, H_{2}, H_{3}\right)$ is the magnetic field, $p$ is the pressure function, $\Psi$ is the Newtonian potential, $G$ is the gravitational constant, and $\mu_{0}$ is the permeability of vacuum.

\subsection{The expression of stationary equation in spherical coordinates}

Now, we will give a new method to get a specific expression of (2.1) in spherical coordinates. Based on a recent paper [14], Wang and Wang gave the general definitions of curl and cross products on a 3-D Riemanion manifold $\left(M, g_{i j}\right)$ with metric

$$
d s^{2}=g_{i j} d x^{i} d x^{j} \quad(i, j=1,2,3) .
$$

Let $A=\left(A^{1}, A^{2}, A^{3}\right)$ and $B=\left(B^{1}, B^{2}, B^{3}\right)$ be smooth vector fields. From [14] we immediately get the following formulae for $\nabla \times A$ and $A \times B$ :

$$
\begin{gathered}
\nabla A=\left(g^{11} \frac{\partial A^{1}}{\partial x^{1}}, g^{22} \frac{\partial A^{2}}{\partial x^{2}}, g^{33} \frac{\partial A^{3}}{\partial x^{3}}\right), \\
\nabla \times A=\frac{1}{\sqrt{g}}\left(\frac{\partial A_{3}}{\partial x^{2}}-\frac{\partial A_{2}}{\partial x^{3}}, \frac{\partial A_{1}}{\partial x^{3}}-\frac{\partial A_{3}}{\partial x^{1}}, \frac{\partial A_{2}}{\partial x^{1}}-\frac{\partial A_{1}}{\partial x^{2}}\right), \\
A \times B=\left(\sqrt{\frac{g_{22} g_{33}}{g_{11}}}\left(A^{2} B^{3}-A^{3} B^{2}\right), \sqrt{\frac{g_{11} g_{33}}{g_{22}}}\left(A^{3} B^{1}-A^{1} B^{3}\right),\right. \\
\left.\sqrt{\frac{g_{11 g_{22}}}{g_{33}}}\left(A^{1} B^{2}-A^{2} B^{1}\right)\right),
\end{gathered}
$$

where $\left(A_{1}, A_{2}, A_{3}\right)=\left(g_{11} A^{1}, g_{22} A^{2}, g_{33} A^{3}\right)$.

Let $x=\left(x_{1}, x_{2}, x_{3}\right) \in \mathbb{R}^{3}, r^{2}=x_{1}^{2}+x_{2}^{2}+x_{3}^{2}$, and $\theta=\arccos \frac{x_{3}}{r}$. In the spherical coordinates, the metric is

$$
d s^{2}=d r^{2}+r^{2} d \theta^{2}+r^{2} \sin ^{2} \theta d \varphi^{2}
$$

Obviously, we have

$$
\left\{\begin{array}{l}
g_{11}=1, \quad g_{22}=r^{2}, \quad g_{33}=r^{2} \sin ^{2} \theta, \\
g_{i j}=0 \quad(i \neq j), \quad g^{k k}=\frac{1}{g_{k k}} \quad(k=1,2,3), \\
\sqrt{g}=r^{2} \sin \theta .
\end{array}\right.
$$

Let $H(x)=H(r, \theta, \varphi)=\left(H^{1}, H^{2}, H^{3}\right)$. Hence, from (2.7) we have

$$
H_{1}=g_{1 j} H^{j}=H^{1}, \quad H_{2}=g_{2 j} H^{j}=r^{2} H^{2}, \quad H_{3}=g_{3 j} H^{j}=r^{2} \sin ^{2} \theta H^{3} .
$$


Using the definition of curl product (2.4), we get

$$
\begin{aligned}
\nabla \times H= & \frac{1}{\sqrt{g}}\left(\frac{\partial H_{3}}{\partial \theta}-\frac{\partial H_{2}}{\partial \varphi}, \frac{\partial H_{1}}{\partial \varphi}-\frac{\partial H_{3}}{\partial r}, \frac{\partial H_{2}}{\partial r}-\frac{\partial H_{1}}{\partial \theta}\right) \\
= & \frac{1}{r^{2} \sin \theta}\left(2 r^{2} \sin \theta \cos \theta H^{3}+r^{2} \sin \theta \frac{\partial H^{3}}{\partial \theta}-r^{2} \frac{\partial H^{2}}{\partial \varphi},\right. \\
& \left.\frac{\partial H^{1}}{\partial \varphi}-2 r \sin ^{2} \theta H^{3}-r^{2} \sin ^{2} \theta \frac{\partial H^{3}}{\partial r}, 2 r \sin \theta H^{2}+r^{2} \frac{\partial H^{2}}{\partial r}-\frac{\partial H^{1}}{\partial \theta}\right) .
\end{aligned}
$$

It follows from the definition of cross product (2.5) and (2.9) that

$$
\begin{aligned}
(\nabla \times & H) \times H \\
= & \left(H^{2} \frac{\partial H^{1}}{\partial \theta}+H^{3} \frac{\partial H^{1}}{\partial \varphi}-2 r\left(H^{2}\right)^{2}-2 r \sin ^{2} \theta\left(H^{3}\right)^{2}-r^{2} H^{2} \frac{\partial H^{2}}{\partial r}-r^{2} \sin ^{2} \theta H^{3} \frac{\partial H^{3}}{\partial r}\right. \\
& H^{1} \frac{\partial H^{2}}{\partial r}+H^{3} \frac{\partial H^{2}}{\partial \varphi}+\frac{2}{r} H^{1} H^{2}-\frac{1}{r^{2}} H^{1} \frac{\partial H^{1}}{\partial \theta}-2 \sin \theta \cos \theta\left(H^{3}\right)^{2}-\sin ^{2} \theta H^{3} \frac{\partial H^{3}}{\partial \theta} \\
& H^{1} \frac{\partial H^{3}}{\partial r}+H^{2} \frac{\partial H^{3}}{\partial \theta}+\frac{2}{r} H^{1} H^{3}+\frac{2 \cos \theta}{\sin \theta} H^{2} H^{3}-\frac{1}{r^{2} \sin ^{2} \theta} H^{1} \frac{\partial H^{1}}{\partial \varphi} \\
& \left.-\frac{1}{\sin ^{2} \theta} H^{2} \frac{\partial H^{2}}{\partial \varphi}\right) .
\end{aligned}
$$

Combining (2.3) and (2.7), it is easy to see that

$$
\begin{aligned}
& \nabla P(\rho)+\rho \nabla \Psi \\
& =\left(\frac{\partial p}{\partial r}+\rho \frac{\partial \Psi}{\partial r}, \frac{1}{r^{2}} \frac{\partial p}{\partial \theta}+\frac{\rho}{r^{2}} \frac{\partial \Psi}{\partial \theta}, \frac{1}{r^{2} \sin ^{2} \theta} \frac{\partial p}{\partial \varphi}+\frac{\rho}{r^{2} \sin ^{2} \theta} \frac{\partial \Psi}{\partial \varphi}\right)
\end{aligned}
$$

Hence, we have, by (2.1), (2.10), and (2.11),

$$
\left\{\begin{aligned}
\frac{\partial p}{\partial r}+\rho \frac{\partial \Psi}{\partial r}= & \mu_{0}\left(H^{2} \frac{\partial H^{1}}{\partial \theta}+H^{3} \frac{\partial H^{1}}{\partial \varphi}-2 r\left(H^{2}\right)^{2}-2 r \sin ^{2} \theta\left(H^{3}\right)^{2}\right. \\
& \left.-r^{2} H^{2} \frac{\partial H^{2}}{\partial r}-r^{2} \sin ^{2} \theta H^{3} \frac{\partial H^{3}}{\partial r}\right) \\
\frac{1}{r^{2}} \frac{\partial p}{\partial \theta}+\frac{\rho}{r^{2}} \frac{\partial \Psi}{\partial \theta}= & \mu_{0}\left(H^{1} \frac{\partial H^{2}}{\partial r}+H^{3} \frac{\partial H^{2}}{\partial \varphi}+\frac{2}{r} H^{1} H^{2}-\frac{1}{r^{2}} H^{1} \frac{\partial H^{1}}{\partial \theta}\right. \\
& \left.-2 \sin \theta \cos \theta\left(H^{3}\right)^{2}-\sin ^{2} \theta H^{3} \frac{\partial H^{3}}{\partial \theta}\right) \\
\frac{1}{r^{2} \sin ^{2} \theta} \frac{\partial p}{\partial \varphi}+\frac{\rho}{r^{2} \sin ^{2} \theta} \frac{\partial \Psi}{\partial \varphi}= & \mu_{0}\left(H^{1} \frac{\partial H^{3}}{\partial r}+H^{2} \frac{\partial H^{3}}{\partial \theta}+\frac{2}{r} H^{1} H^{3}\right. \\
& \left.+\frac{2 \cos \theta}{\sin \theta} H^{2} H^{3}-\frac{1}{r^{2} \sin ^{2} \theta} H^{1} \frac{\partial H^{1}}{\partial \varphi}-\frac{1}{\sin ^{2} \theta} H^{2} \frac{\partial H^{2}}{\partial \varphi}\right)
\end{aligned}\right.
$$

Let $H_{r}=H^{1}, H_{\theta}=\frac{H^{2}}{r}, H_{\varphi}=\frac{H^{3}}{r \sin \theta}$. The spherical coordinate expressions of (2.12) can be rewritten as follows:

$$
\left\{\begin{aligned}
\frac{\partial p}{\partial r}+\rho \frac{\partial \Psi}{\partial r}= & \mu_{0}\left(\frac{H_{\theta}}{r} \frac{\partial H_{r}}{\partial \theta}+\frac{H_{\varphi}}{r \sin \theta} \frac{\partial H_{r}}{\partial \varphi}-\frac{H_{\theta}^{2}+H_{\varphi}^{2}}{r}\right. \\
& \left.-H_{\theta} \frac{\partial H_{\theta}}{\partial r}-H_{\varphi} \frac{\partial H_{\varphi}}{\partial r}\right), \\
\frac{1}{r} \frac{\partial p}{\partial \theta}+\frac{\rho}{r} \frac{\partial \Psi}{\partial \theta}= & \mu_{0}\left(H_{r} \frac{\partial H_{\theta}}{\partial r}+\frac{H_{\varphi}}{r \sin \theta} \frac{\partial H_{\theta}}{\partial \varphi}+\frac{H_{r} H_{\theta}}{r}-\frac{\cos \theta}{r \sin \theta} H_{\varphi}^{2}\right. \\
& \left.\quad-\frac{1}{r} H_{r} \frac{\partial H_{r}}{\partial \theta}-\frac{1}{r} H_{\varphi} \frac{\partial H_{\varphi}}{\partial \theta}\right), \\
\frac{1}{r \sin \theta} \frac{\partial p}{\partial \varphi}+\frac{\rho}{r \sin \theta} \frac{\partial \Psi}{\partial \varphi}= & \mu_{0}\left(H_{r} \frac{\partial H_{\varphi}}{\partial r}+\frac{H_{\theta}}{r} \frac{\partial H_{\varphi}}{\partial \theta}+\frac{H_{r} H_{\varphi}}{r}\right. \\
& \left.+\frac{\cos \theta}{r \sin \theta} H_{\theta} H_{\varphi}-\frac{1}{r \sin \theta} H_{r} \frac{\partial H_{r}}{\partial \varphi}-\frac{1}{r \sin \theta} H_{\theta} \frac{\partial H_{\theta}}{\partial \varphi}\right) .
\end{aligned}\right.
$$


Remark 2.1 Let $H=\left(H_{r}, H_{\theta}, H_{\varphi}\right)$. Notice that (2.13) can be directly calculated by the definitions of gradient and of curl and cross products in spherical coordinates as follows:

$$
\begin{gathered}
\nabla=\left(\frac{\partial}{\partial r}, \frac{1}{r} \frac{\partial}{\partial \theta}, \frac{1}{r \sin \theta} \frac{\partial}{\partial \varphi}\right), \\
\nabla \cdot H=\frac{1}{r^{2}} \frac{\partial\left(r^{2} H_{r}\right)}{\partial r}+\frac{1}{r \sin \theta} \frac{\partial\left(\sin \theta H_{\theta}\right)}{\partial \theta}+\frac{1}{r \sin \theta} \frac{\partial H_{\varphi}}{\partial \varphi}, \\
\nabla \times H=\frac{1}{r^{2} \sin \theta}\left(r \cos \theta H_{\varphi}+r \sin \theta \frac{\partial H_{\varphi}}{\partial \theta}-r \frac{\partial H_{\theta}}{\partial \varphi},\right. \\
r \frac{\partial H_{r}}{\partial \varphi}-r \sin \theta H_{\varphi}-r^{2} \sin \theta \frac{\partial H_{\varphi}}{\partial r}, \\
\left.r \sin \theta H_{\theta}+r^{2} \sin \theta \frac{\partial H_{\theta}}{\partial r}-r \sin \theta \frac{\partial H_{r}}{\partial \theta}\right) .
\end{gathered}
$$

However, we give a new method to get (2.13) by using the definitions of curl and cross products (2.4) and (2.5) on the 3-D Riemanion manifold $M$, which only need a simple transformation $\left(H_{r}, H_{\theta}, H_{\varphi}\right)=\left(H^{1}, \frac{H^{2}}{r}, \frac{H^{3}}{r \sin \theta}\right)$.

Noticing that the solutions we look for have the form (1.5) and omitting the terms $\frac{\partial}{\partial \varphi}$, we can rewrite (2.13) as follows:

$$
\left\{\begin{array}{l}
\frac{\partial p}{\partial r}+\rho \frac{\partial \Psi}{\partial r}=\mu_{0}\left(\frac{H_{\theta}}{r} \frac{\partial H_{r}}{\partial \theta}-\frac{H_{\theta}^{2}}{r}-H_{\theta} \frac{\partial H_{\theta}}{\partial r}\right), \\
\frac{1}{r} \frac{\partial p}{\partial \theta}+\frac{\rho}{r} \frac{\partial \Psi}{\partial \theta}=\mu_{0}\left(H_{r} \frac{\partial H_{\theta}}{\partial r}+\frac{H_{r} H_{\theta}}{r}-\frac{1}{r} H_{r} \frac{\partial H_{r}}{\partial \theta}\right) .
\end{array}\right.
$$

Let $H_{\varphi}=0$. Omitting the terms $\frac{\partial}{\partial \varphi}$ in (2.15), it is easy to see that $\nabla \cdot H=0$ implies that

$$
r^{2} \frac{\partial H_{r}}{\partial r}+2 r H_{r}+r \frac{\partial H_{\theta}}{\partial \theta}+r \frac{\cos \theta}{\sin \theta} H_{\theta}=0 .
$$

For simplicity, we denote

$$
m=\left(\frac{\partial H_{\theta}}{\partial r}+\frac{H_{\theta}}{r}-\frac{1}{r} \frac{\partial H_{r}}{\partial \theta}\right) .
$$

Then (2.17) can be rewritten as

$$
\left\{\begin{array}{l}
\frac{\partial p}{\partial r}+\rho \frac{\partial \Psi}{\partial r}=-\mu_{0} m H_{\theta}, \\
\frac{1}{r} \frac{\partial p}{\partial \theta}+\frac{\rho}{r} \frac{\partial \Psi}{\partial \theta}=\mu_{0} m H_{r} .
\end{array}\right.
$$

\subsection{The problem and formulation}

Owing to (2.18), we get

$$
\frac{\partial\left(r^{2} \sin \theta H_{r}\right)}{\partial r}+\frac{\partial\left(r \sin \theta H_{\theta}\right)}{\partial \theta}=0,
$$

which enables us to introduce a magnetic potential $\varphi_{0}(r, \theta)$ such that

$$
\left\{\begin{array}{l}
\frac{\partial \varphi_{0}}{\partial r}=r \sin \theta H_{\theta} \\
\frac{\partial \varphi_{0}}{\partial \theta}=-r^{2} \sin \theta H_{r} .
\end{array}\right.
$$


Let

$$
n(\rho)=\int_{0}^{\rho} \frac{p^{\prime}(s)}{s} d s .
$$

Then (2.19) and (2.21) imply

$$
\left\{\begin{array}{l}
\rho \frac{\partial(n(\rho)+\Psi)}{\partial r}=-\mu_{0} m\left(\frac{1}{r \sin \theta} \frac{\partial}{\partial} \varphi_{0}\right), \\
\rho \frac{\partial(n(\rho)+\Psi)}{\partial \theta}=-\mu_{0} m\left(\frac{1}{r \sin \theta} \frac{\partial}{\partial \theta} \varphi_{0}\right) .
\end{array}\right.
$$

Let

$$
\frac{\mu_{0} m}{\rho r \sin \theta}=W .
$$

In this paper, we only consider the case that $W$ is a constant. Then it follows from (2.23) and (2.24) that

$$
\left\{\begin{array}{l}
\frac{\partial}{\partial r}(n(\rho)+\Psi)=-W \frac{\partial}{\partial r} \varphi_{0}, \\
\frac{\partial}{\partial \theta}(n(\rho)+\Psi)=-W \frac{\partial}{\partial \theta} \varphi_{0} .
\end{array}\right.
$$

Note that (2.25) implies that

$$
\nabla\left(n(\rho)+\Psi+W \varphi_{0}\right)=0 \quad \text { whenever } \rho>0 .
$$

Therefore,

$$
n(\rho)+\Psi+W \varphi_{0}=\text { const. }:=\lambda, \quad \text { in the region } \rho>0,
$$

where $n(\rho)$ is given by (2.22), and $\Psi$ is given by (1.3).

In the following, we only need to solve problem (2.27) with the total mass constraint

$$
\int_{\mathbb{R}^{3}} \rho(x) d x=M>0 \quad \text { for some given } M \text {. }
$$

\section{Existence of star solution coupling to a magnetic field}

\subsection{The expression of magnetic potential}

Combining (2.14) and $\frac{\partial}{\partial \varphi}=0$, we have

$$
\nabla \varphi_{0}=\left(\frac{\partial \varphi_{0}}{\partial r}, \frac{1}{r} \frac{\partial \varphi_{0}}{\partial \theta}, \frac{1}{r \sin \theta} \frac{\partial \varphi_{0}}{\partial \varphi}\right)=\left(\frac{\partial \varphi_{0}}{\partial r}, \frac{1}{r} \frac{\partial \varphi_{0}}{\partial \theta}, 0\right) .
$$

Using the divergence formula (2.15), from (2.21), (2.24), and (3.1) we can deduce that

$$
\operatorname{div}\left(\frac{1}{r^{2} \sin ^{2} \theta} \nabla \varphi_{0}\right)=\frac{m}{r \sin \theta}=\frac{W}{\mu_{0}} \rho .
$$

Let

$$
\varphi_{0}\left(x_{1}, x_{2}, x_{3}\right)=\varphi_{0}(r, \theta)
$$


Then (3.2) is equivalent to

$$
\frac{1}{x_{1}^{2}+x_{2}^{2}}\left(\frac{\partial^{2} \varphi_{0}}{\partial x_{1}^{2}}+\frac{\partial^{2} \varphi_{0}}{\partial x_{2}^{2}}+\frac{\partial^{2} \varphi_{0}}{\partial x_{3}^{2}}-\frac{2 x_{1}}{x_{1}^{2}+x_{2}^{2}} \frac{\partial \varphi_{0}}{\partial x_{1}}-\frac{2 x_{2}}{x_{1}^{2}+x_{2}^{2}} \frac{\partial \varphi_{0}}{\partial x_{2}}\right)=\frac{W}{\mu_{0}} \rho,
$$

where

$$
\rho\left(x_{1}, x_{2}, x_{3}\right)=\rho(r, \theta) .
$$

Define the operator $\mathcal{L}$ as follows:

$$
\mathcal{L}=\frac{1}{x_{1}^{2}+x_{2}^{2}}\left(\frac{\partial^{2}}{\partial x_{1}^{2}}+\frac{\partial^{2}}{\partial x_{2}^{2}}+\frac{\partial^{2}}{\partial x_{3}^{2}}-\frac{2 x_{1}}{x_{1}^{2}+x_{2}^{2}} \frac{\partial}{\partial x_{1}}-\frac{2 x_{2}}{x_{1}^{2}+x_{2}^{2}} \frac{\partial}{\partial x_{2}}\right) .
$$

It is easy to observe that $\mathcal{L}$ is a symmetric operator. Hence, it has a Green's function $G(x, y)$ for $x, y \in \mathbb{R}^{3}$. The following lemma gives an exact expression of $G(x, y)$.

Lemma 3.1 The Green's function $G(x, y)$ for $\mathcal{L}$ has the expression

$$
G(x, y)=-\frac{\Lambda\left(\left(x_{1}-y_{1}\right)^{2}+\left(x_{2}-y_{2}\right)^{2}\right)}{\left(\left(x_{1}-y_{1}\right)^{2}+\left(x_{2}-y_{2}\right)^{2}+\left(x_{3}-y_{3}\right)^{2}\right)^{\frac{3}{2}}},
$$

where is a positive constant.

Proof We know that (3.2) is equivalent to (3.4). Let $\varphi_{0}=r^{2} \bar{\varphi} \sin ^{2} \theta$. Inserting this into (3.2), we get

$$
\frac{\partial^{2} \bar{\varphi}}{\partial r^{2}}+\frac{4}{r} \frac{\partial \bar{\varphi}}{\partial r}+\frac{3 \cos \theta}{r^{2} \sin \theta} \frac{\partial \bar{\varphi}}{\partial \theta}+\frac{1}{r^{2}} \frac{\partial^{2} \bar{\varphi}}{\partial \theta^{2}}=\frac{W \rho}{\mu_{0}},
$$

and it is not difficult to observe that the Green's function of the operator

$$
\frac{\partial^{2}}{\partial r^{2}}+\frac{4}{r} \frac{\partial}{\partial r}+\frac{3 \cos \theta}{r^{2} \sin \theta} \frac{\partial}{\partial \theta}+\frac{1}{r^{2}} \frac{\partial^{2}}{\partial \theta^{2}}
$$

is given by

$$
G(r, \theta)=-\frac{\Lambda}{r^{3}}
$$

where $\Lambda$ is a positive constant.

Hence, the Green's function $G(x, y)$ of $\mathcal{L}$ can be expressed as

$$
G(x, y)=-\frac{\Lambda r^{2} \sin ^{2} \theta}{r^{3}}=\frac{\Lambda\left(\left(x_{1}-y_{1}\right)^{2}+\left(x_{2}-y_{2}\right)^{2}\right)}{\left(\left(x_{1}-y_{1}\right)^{2}+\left(x_{2}-y_{2}\right)^{2}+\left(x_{3}-y_{3}\right)^{2}\right)^{\frac{3}{2}}} .
$$

\subsection{Variational formulation}

In this paper, we assume that the pressure function $p(\rho)$ satisfies the $\alpha$-law (1.2) for some constant $\alpha>1$. Let

$$
I(\rho)=\frac{\rho^{\alpha}}{\alpha-1} .
$$


Then

$$
n(\rho)=I^{\prime}(\rho)
$$

Moreover, the Newtonian potential operator is given by (1.3), and we can denote

$$
\Psi(x)=-\int_{\mathbb{R}^{3}} \frac{\rho(y)}{|x-y|} d y:=-E(\rho) .
$$

Notice that the magnetic potential $\varphi_{0}$ satisfies (3.2). By Lemma 3.1 we see that $G(x, y)$ for $x, y \in \mathbb{R}^{3}$ is the Green's function for the operator $\mathcal{L}$ defined in (3.5), that is,

$$
\mathcal{L} G=\delta(x-y)
$$

where $\delta(x-y)$ is the Dirac measure giving the unit mass to the point $x$. Since $\mathcal{L}$ is symmetric, we have

$$
\left\langle\mathcal{L} \varphi_{0}, G\right\rangle=\left\langle\varphi_{0}, \mathcal{L} G\right\rangle=\left\langle\varphi_{0}, \delta(x-y)\right\rangle=\varphi_{0}(y)
$$

where the inner product $\langle\cdot, \cdot\rangle$ is taken in $L^{2}$. Thus, we have the following integral representation for $\varphi_{0}$ :

$$
\varphi_{0}(x)=D(\rho)
$$

where the integral operator $D$ is given by

$$
D(\rho)=\frac{W}{\mu_{0}} \int_{\mathbb{R}^{3}} G(x, y) \rho(y) d y .
$$

By (3.11), (3.12), and (3.16) it is obvious that equation (2.27) can be written as

$$
I^{\prime}(\rho)+E(\rho)+W D(\rho)=\lambda \quad \text { for } \rho>0 .
$$

According to (3.17), we define the energy functional $F$ as follows:

$$
F(\rho)=\int_{\mathbb{R}^{3}}\left[I(\rho)+\frac{1}{2} \rho E(\rho)+\frac{1}{2} \rho W D(\rho)\right] d x,
$$

where $I(\rho)$ is the function given in (3.10). The energy functional $F(\rho)$ means that solving (3.17) with the total mass constraint (2.28) is equivalent to proving that (3.18) has a minimizer in some function space $X$.

Now, we review the results for stationary solution (1.5). For $0<M<\infty$, we define $X_{M}$ by

$$
\begin{aligned}
X_{M}= & \left\{\rho: \mathbb{R}^{3} \rightarrow \mathbb{R}, \rho>0 \text { a.e., } \int_{\mathbb{R}^{3}} \rho d x=M\right. \text {, and } \\
& \left.\int_{\mathbb{R}^{3}}\left[I(\rho)+\frac{1}{2} \rho E(\rho)\right] d x<\infty\right\} .
\end{aligned}
$$


For $\rho \in X_{M}$, we define the energy function $F_{1}$ for the nonrotating nonmagnetic by

$$
F_{1}(\rho)=\int_{\mathbb{R}^{3}}\left[I(\rho)+\frac{1}{2} \rho E(\rho)\right] d x .
$$

Thanks to the Lemma 2 in [1], Federbush et al. [4] obtained the following useful lemma for the minimizer of the functional $F_{1}$.

Lemma 3.2 Suppose that the pressure $p(\rho)=\rho^{\alpha}\left(\alpha>\frac{4}{3}\right)$. Let $\rho^{*}$ be a minimizer of (3.20) in $X_{M}$, and let

$$
\Gamma_{M}=\left\{x \in \mathbb{R}^{3}: \rho^{*}>0\right\} .
$$

Then there exists a constant $\lambda_{1}$ such that

$$
\left\{\begin{array}{l}
I(\rho)+\frac{1}{2} \rho E(\rho)=\lambda_{1}, \quad x \in \Gamma_{M} \\
E(\rho) \geq \lambda_{1}, \quad x \in \mathbb{R}-\Gamma_{M} .
\end{array}\right.
$$

Remark 3.3 The variational problem is unusual, in that a solution turns out to have compact support. The reason is that the functional one seeks to minimize is not lower semicontinuous on the class of all admissible functions. Auchmuty and Richard [1] first restrict their considerations to functions with support in the ball of radius $R_{M}$. Hence, we should find the radius $R_{M}$. Note that, for $\alpha>\frac{4}{3}$, Luo and Smoller [8] have proved that a local minimizer $\rho^{*}$ of the function $F_{1}$ in $X_{M}$ exists. Also, they showed that the minimizer $\rho^{*}$ is actually radial and unique and has compact support, that is, for given total mass $M$, there exists a unique constant $R_{M}>0$ such that

$$
\begin{cases}\rho^{*}(x)>0 & \text { if }|x|<R_{M} \\ \rho^{*}(x)=0 & \text { if }|x| \geq R_{M} .\end{cases}
$$

Notice that $\rho^{*}$ satisfying (3.22) in $X_{M}$ is called a nonrotating nonmagnetic star solution, and $R_{M}$ is called the radius of the nonrotating nonmagnetic star solution with total mass $M$.

Based on (3.23), we define the function spaces $Y_{M}$ and $Y_{M R_{0}}^{\alpha}$ as follows:

$$
\begin{aligned}
Y_{M}^{\alpha}= & \left\{\rho: \mathbb{R}^{3} \rightarrow \mathbb{R}, \rho(x)=\rho(r, \theta), \rho \geq 0 \text {, a.e., } \rho \in L^{1}\left(\mathbb{R}^{3}\right) \cap L^{\alpha}\left(\mathbb{R}^{3}\right),\right. \\
& \left.\alpha>\frac{4}{3}, \int_{\mathbb{R}^{3}} \rho(x) d x=M\right\}, \\
Y_{M R_{0}}^{\alpha}= & \left\{\rho \in Y_{M}^{\alpha}, \rho=0 \text { for } r \geq R_{0}\right\},
\end{aligned}
$$

where $r=\sqrt{x_{1}^{2}+x_{2}^{2}+x_{3}^{2}}, \theta=\arccos \frac{x_{3}}{r}, R_{0} \geq R_{M}$ is a constant, and $R_{M}$ is the radius of the nonrotating nonmagnetic star solution with prescribed total mass $M$.

We want to apply Theorem 3.2 in [4]. It is easy to see that a minimizer of the functional $F$ as defined in(3.18) in $Y_{M R_{0}}^{\alpha}$ solves equation (3.17). 
Theorem 3.4 Let $\rho_{1}^{*}$ be a minimizer of the energy functional $F$ in $Y_{M R_{0}}^{\alpha}$, and

$$
\Gamma_{M}=\left\{x \in \mathbb{R}^{3}: \rho_{1}^{*}(x)>0\right\} .
$$

If $\alpha>\frac{6}{5}$, then $\rho_{1}^{*} \in C\left(\mathbb{R}^{3}\right) \cap C^{1}\left(\Gamma_{M}\right)$. Moreover, there exists a constant $\lambda_{M}^{*}$ such that

$$
I^{\prime}\left(\rho_{1}^{*}\right)+E\left(\rho_{1}^{*}\right)+W D\left(\rho_{1}^{*}\right)=\lambda_{M}^{*}, \quad x \in \Gamma_{M} .
$$

Proof Let

$$
F_{2}(\rho)=\frac{1}{2} W \int_{\mathbb{R}^{3}} \rho D(\rho) d x .
$$

Then $F(\rho)$ can be written in two parts:

$$
F(\rho)=F_{1}(\rho)+F_{2}(\rho)
$$

where $F_{1}(\rho)$ is defined by (3.20).

Let $\rho+t \sigma \in Y_{M R_{0}}^{\alpha}$ for any $t \in \mathbb{R}$ under the condition $\int_{\mathbb{R}^{3}} \sigma d x=0$. Let us note carefully that

$$
\begin{aligned}
\lim _{t \rightarrow 0} & \frac{F_{1}(\rho+t \sigma)-F_{1}(\rho)}{t} \\
= & \lim _{t \rightarrow 0} \frac{1}{t} \int_{\mathbb{R}^{3}}\left(I(\rho+t \sigma)+\frac{1}{2}(\rho+t \sigma) E(\rho+t \sigma)-I(\rho)-\frac{1}{2} \rho E(\rho)\right) d x \\
= & \lim _{t \rightarrow 0} \int_{\mathbb{R}^{3}} \frac{I(\rho+t \sigma)-I(\rho)}{t} d x+\lim _{t \rightarrow 0} \int_{\mathbb{R}^{3}} \frac{\rho E(\rho+t \sigma)-\rho E(\rho)}{2 t} d x \\
& +\int_{\mathbb{R}^{3}} \frac{1}{2} \sigma E(\rho) d x \\
= & \int_{\mathbb{R}^{3}} I^{\prime}(\rho) \sigma d x+\lim _{t \rightarrow 0} \frac{1}{t} \int_{\mathbb{R}^{3}} \frac{\rho}{2}\left(\int_{\mathbb{R}^{3}} \frac{-\rho-t \sigma}{|x-y|} d y+\int_{\mathbb{R}^{3}} \frac{\rho}{|x-y|} d y\right) d x \\
& +\int_{\mathbb{R}^{3}} \frac{1}{2} \sigma E(\rho) d x \\
= & \int_{\mathbb{R}^{3}}\left(I^{\prime}(\rho) \sigma+\frac{1}{2} \sigma E(\rho)\right) d x+\int_{\mathbb{R}^{3}} \frac{\sigma(y)}{2}\left(\int_{\mathbb{R}^{3}} \frac{-\rho(x)}{|x-y|} d x\right) d y \\
= & \int_{\mathbb{R}^{3}}\left(I^{\prime}(\rho)+E(\rho)\right) \sigma d x
\end{aligned}
$$

if $\rho \in Y_{M R_{0}}^{\alpha}$.

For $F_{2}(\rho)$, we get

$$
\begin{aligned}
\lim _{t \rightarrow 0} & \frac{F_{1}(\rho+t \sigma)-F_{1}(\rho)}{t} \\
= & \lim _{t \rightarrow 0} \frac{1}{t} \int_{\mathbb{R}^{3}}\left(\frac{W}{2}(\rho+t \sigma) D(\rho+t \sigma)-\frac{W}{2} \rho D(\rho)\right) d x \\
= & \lim _{t \rightarrow 0} \frac{1}{t} \int_{\mathbb{R}^{3}} \frac{W \rho}{2}(D(\rho+t \sigma)-D(\rho)) d x+\int_{\mathbb{R}^{3}} \frac{W \sigma}{2} D(\rho) d x \\
= & \lim _{t \rightarrow 0} \int_{\mathbb{R}^{3}} \frac{W \rho}{2 t}\left(\int_{\mathbb{R}^{3}}(G(x, y)(\rho+t \sigma)-G(x, y) \rho) d y\right) d x+\int_{\mathbb{R}^{3}} \frac{W \sigma}{2} D(\rho) d x
\end{aligned}
$$




$$
\begin{aligned}
& =\int_{\mathbb{R}^{3}} \frac{W \rho}{2}\left(\int_{\mathbb{R}^{3}} G(x, y) \sigma d y\right) d x+\int_{\mathbb{R}^{3}} \frac{W \sigma}{2} D(\rho) d x \\
& =\int_{\mathbb{R}^{3}} \frac{W \sigma}{2}\left(\int_{\mathbb{R}^{3}} G(x, y) \rho(x) d x\right) d y+\int_{\mathbb{R}^{3}} \frac{W \sigma}{2} D(\rho) d x \\
& =\int_{\mathbb{R}^{3}} W \sigma D(\rho) d x,
\end{aligned}
$$

where $G(x, y)$ is defined by (3.6).

Hence, from (3.30) and (3.31) we have

$$
\begin{aligned}
& \lim _{t \rightarrow 0} \frac{F(\rho+t \sigma)-F(\rho)}{t} \\
& \quad=\int_{\mathbb{R}^{3}}\left[I^{\prime}(\rho)+E(\rho)+D(\rho)\right] \sigma d x=0
\end{aligned}
$$

for all $\sigma$ satisfying $\int_{\mathbb{R}^{3}} \sigma d x=0$. Then we can prove the theorem using a similar argument as in [1].

Now, we give the main theorem of this paper.

Theorem 3.5 Suppose that $\alpha>\frac{4}{3}$. Then the following statements hold:

1. $\inf _{Y_{M R_{0}}^{\alpha}} F(\rho)<0$,

2. $\quad F(\rho) \geq C_{1} \int_{\mathbb{R}^{3}} \rho^{\alpha} d x-C_{2}, \rho \in Y_{M R_{0}}^{\alpha}$, for some positive constants $C_{1}$ and $C_{2}$ independent of $\rho$,

3. F has a minimizer $\rho^{*}$ in $Y_{M R_{0}}^{\alpha}$.

Remark 3.6 In comparison with Theorem 3.3 with adiabatic exponent $\alpha>2$ in [4], we only need the adiabatic exponent $\alpha>\frac{4}{3}$.

\subsection{The proof of Theorem 3.5}

Before giving the proof of Theorem 3.5, we introduce the following lemma, which ensures that the functional $F$ is bounded on the set $Y_{M R_{0}}^{\alpha}$ if $\alpha>\frac{4}{3}$.

Let

$$
F_{M}=\inf _{\rho \in Y_{M R_{0}}^{\alpha}} F(\rho)
$$

Lemma 3.7 Let $\alpha>\frac{4}{3}$. If $\rho \in Y_{M R_{0}}^{\alpha}$, then there exist two positive constants $C_{1}$ and $C_{2}$ depending only on $\alpha$ and $M$ such that

$$
C_{1} \int_{\mathbb{R}^{3}} \rho^{\alpha} d x-C_{2}<F(\rho) \text { and } F_{M}<0 .
$$

Proof Let $F(\rho)$ be defined by (3.29). For $F_{1}(\rho)$, Lemma 2.4 in [8] implies that $C_{1} \int_{\mathbb{R}^{3}} \rho^{\alpha} d x-$ $C_{2}<F_{1}(\rho)$ for two positive constants $C_{1}$ and $C_{2}$ depending only on $\alpha$ and $M$. Here, we only prove that $C_{1} \int_{\mathbb{R}^{3}} \rho^{\alpha} d x-C_{2}<F_{2}(\rho)$. By Hölder's inequality we have

$$
\begin{aligned}
F_{2}(\rho) & =\frac{1}{2} W \int_{\mathbb{R}^{3}} \rho D(\rho) d x \\
& \leq C\|\rho\|_{2-\varepsilon}\|D(\rho)\|_{\frac{2-\varepsilon}{1-\varepsilon}} .
\end{aligned}
$$


Note that

$$
D(\rho)=-\frac{\Lambda W}{\mu_{0}} \int_{\mathbb{R}^{3}} \frac{\left(x_{1}-y_{1}\right)^{2}+\left(x_{2}-y_{2}\right)^{2}}{\left(\left(x_{1}-y_{1}\right)^{2}+\left(x_{2}-y_{2}\right)^{2}+\left(x_{3}-y_{3}\right)^{2}\right)^{\frac{3}{2}}} \rho(y) d y .
$$

By the Riesz potential estimate [16], Lemma 7.12, p.159, we get

$$
\|D(\rho)\|_{\frac{2-\varepsilon}{1-\varepsilon}} \leq C|\Omega|^{\mu-\delta}\|\rho\|_{p} \quad \text { if } p<\frac{3(2-\varepsilon)}{7-5 \varepsilon}
$$

for $\mu=\frac{2}{3}$ and $\delta=\frac{1}{p}-\frac{1-\varepsilon}{2-\varepsilon}$, where $\Omega$ is the compact support of $\rho$.

By the interpolation inequality (Theorem 2.11 in [17]), if $f \in L^{q} \cap L^{r}(1 \leq q<p<r<\infty)$, then

$$
\|f\|_{p} \leq\|f\|_{q}^{a}\|f\|_{r}^{1-a}
$$

for $a=\frac{p^{-1}-r^{-1}}{q^{-1}-r^{-1}}$.

Inserting $q=1, r=2-\varepsilon$, and $a=\frac{(2-\varepsilon) / p-1}{1-\varepsilon}<1$ into (3.34), we get that (3.33) implies

$$
\left|\frac{1}{2} \int_{\mathbb{R}^{3}} \rho D(\rho) d x\right| \leq C\|\rho\|_{2-\varepsilon}^{2-a}\|\rho\|_{1}^{a} .
$$

By the interpolation inequality [16], (7.6), p.145, we obtain

$$
\int_{\mathbb{R}^{3}} \rho^{2-\varepsilon} d x \leq \omega \int_{\mathbb{R}^{3}} \rho^{\alpha} d x+\omega^{-\frac{2-\varepsilon}{\alpha-2+\varepsilon}}|\Omega|^{\frac{\alpha-2+\varepsilon}{\alpha}} \leq \omega \int_{\mathbb{R}^{3}} \rho^{\alpha} d x+\omega^{-\frac{2-\varepsilon}{\alpha-2+\varepsilon}}|\Omega|^{\frac{\alpha-2+\varepsilon}{\alpha}}
$$

where $(\alpha>2-\varepsilon)$, and $\Omega$ is the compact support of $\rho$.

Together (3.35) with (3.36), we have

$$
\left|\frac{1}{2} \int_{\mathbb{R}^{3}} \rho D(\rho) d x\right| \leq\|\rho\|_{1}^{a}\left(\int_{\mathbb{R}^{3}} \omega \rho^{\alpha} d x+C(\omega)\right)^{\frac{2-a}{2-\varepsilon}}
$$

where $C(\lambda)=\lambda^{-\frac{2-\varepsilon}{\alpha-2+\varepsilon}}|\Omega|^{\frac{\alpha-2+\varepsilon}{\alpha}}$.

Choosing $a=\varepsilon$, it is obvious that

$$
p=\frac{2-\varepsilon}{1+\varepsilon-\varepsilon^{2}}>\frac{3(2-\varepsilon)}{7-5 \varepsilon},
$$

which implies that $7-5 \varepsilon>3+3 \varepsilon-3 \varepsilon^{2}, \varepsilon<\frac{2}{3}$.

It follows from $\alpha>2-\varepsilon$ that

$$
\alpha>\frac{4}{3} \Longleftrightarrow \varepsilon<\frac{2}{3} .
$$

Hence, for $\alpha>\frac{4}{3}$, we have

$$
\left|\frac{1}{2} \int_{\mathbb{R}^{3}} \rho D(\rho) d x\right| \leq \omega M^{a} \int_{\mathbb{R}^{3}} \rho^{\alpha} d x+C(\omega) M^{a} .
$$


Letting $\omega$ be sufficiently small, then we have

$$
F(\rho)>C \int_{\mathbb{R}^{3}} \rho^{\alpha} d x-C_{1} .
$$

Proof of Theorem 3.5 Note that Lemma 3.7 proves conclusion (2) of Theorem 3.5. Conclusion (3) in Theorem 3.5 can be proved by using the same method as in [8].

Notice that

$$
\begin{aligned}
F_{2}(\rho) & =-\frac{1}{2} W \int_{\mathbb{R}^{3}} \rho D(\rho) d x \\
& =-\frac{\Lambda W^{2}}{\mu_{0}} \rho \int_{\mathbb{R}^{3}} \frac{\left(x_{1}-y_{1}\right)^{2}+\left(x_{2}-y_{2}\right)^{2}}{\left(\left(x_{1}-y_{1}\right)^{2}+\left(x_{2}-y_{2}\right)^{2}+\left(x_{3}-y_{3}\right)^{2}\right)^{\frac{3}{2}}} \rho(y) d y<0 .
\end{aligned}
$$

Also, by the argument in [8] we get

$$
F_{1}(\rho)=\int_{\mathbb{R}^{3}}\left[I(\rho)+\frac{1}{2} \rho E(\rho)\right] d x<0 .
$$

Hence, conclusion (1) of Theorem 3.5 is established.

\section{Competing interests}

The authors declare that they have no competing interests.

\section{Authors' contributions}

All authors contributed equally and significantly in writing this article. All authors read and approved the final manuscript.

\section{Acknowledgements}

The authors are very appreciated to the reviewer for his/her valuable suggestions. Supported by NSFC (11401479).

Received: 27 July 2016 Accepted: 18 November 2016 Published online: 05 December 2016

\section{References}

1. Auchmuty, G, Beals, R: Variational solutions of some nonlinear free boundary problems. Arch. Ration. Mech. Anal. 43, 255-271 (1971)

2. Cai, H, Tan, Z: Existence and stability of stationary solutions to the compressible Navier-Stokes-Poisson equations. Nonlinear Anal., Real World Appl. 32, 260-293 (2016)

3. Chanillo, S, Li, YY: On diameters of uniformly rotating stars. Commun. Math. Phys. 166(2), 417-430 (1994)

4. Federbush, P, Luo, T, Smoller, J: Existence of magnetic compressible fluid stars. Arch. Ration. Mech. Anal. 215(2), 611-631 (2015)

5. Guo, Y, Rein, G: Stable steady states in stellar dynamics. Arch. Ration. Mech. Anal. 147, 225-243 (1999)

6. Jang, J: Nonlinear instability in gravitational Euler-Poisson systems for $\gamma=\frac{6}{5}$. Arch. Ration. Mech. Anal. 188(2), 265-307 (2008)

7. Lieb, EH, Yau, HT: The Chandrasekhar theory of stellar collapse as the limit of quantum mechanics. Commun. Math. Phys. 112(1), 147-174 (1987)

8. Luo, T, Smoller, J: Existence and non-linear stability of rotating star solutions of the compressible Euler-Poisson equations. Arch. Ration. Mech. Anal. 191(3), 447-496 (2009)

9. Rein, G: Non-linear stability of gaseous stars. Arch. Ration. Mech. Anal. 168(2), 115-130 (2003)

10. Kobayashi, T, Suzuki, T: Weak solutions to the Navier-Stokes-Poisson equation. Adv. Math. Sci. Appl. 18, 141-168 (2008)

11. Feireisl, E, Novotný, A, Petleltová, H: On the existence of globally defined weak solutions to the Navier-Stokes equations of isentropic compressible fluids. J. Math. Fluid Mech. 3, 358-392 (2001)

12. Lions, PL: Mathematical Topics in Fluids Mechanics. Clarendon, New York (1998)

13. Luo, T, Xin, ZP, Zeng, HH: On nonlinear asymptotic stability of the Lane-Emden solutions for the viscous gaseous star problem. Adv. Math. 291, 90-182 (2016)

14. Wang, Q, Wang, HC: The dynamical mechanism of jets for AGN. Discrete Contin. Dyn. Syst. 21(3), $943-957$ (2016)

15. Lin, SS: Stability of gaseous stars in spherically symmetric motions. SIAM J. Math. Anal. 28(3), 539-569 (1997)

16. Gilbarg, D, Trudinger, NS: Elliptic Partial Differential Equations of Second Order. Classics in Mathematics. Springer, Berlin (2001) Reprint of the 1998 edition

17. Adams, RA, Fournier, JJF: Sobolev Spaces, 2nd edn. Elsevier, Amsterdam (2003) 